\title{
DE VUELTA AL FIN DE LA HISTORIA. UNA INTERPRETACIÓN ÉTICA DE LA CONDICIÓN POSTHISTÓRICA EN ALEXANDRE KOJĖVE ${ }^{1}$
}

\author{
ORIOL FARRÉS JUSTE \\ Universidad Autónoma de Barcelona
}

\begin{abstract}
RESUMEN: El siguiente trabajo tiene como objetivo analizar e interpretar la teoría del fin de la historia en Alexandre Kojève. El contexto de esta investigación se basa fundamentalmente en la nota a pie de la página añadida por Kojève en la segunda edición (1968) de su libro Introduction à la lecture de Hegel. Esta nota a pie de página ha dado lugar a intensos debates e interrogantes filosóficos importantes. En ella, se toma como paradigma el esnobismo japonés como alternativa a la animalización definitiva del hombre posthistórico. En el trabajo, concretamente, se valorará la rectificación kojeviana sobre el fin de la historia a la luz de la obra de Kojève en su conjunto, sus debates con autores de su tiempo y las referencias a la tradición filosófica. Todo ello con la finalidad de desentrañar las implicaciones éticas de la supervivencia de lo humano en el fin de la historia y su relación con los planteamientos postmetafísicos contemporáneos.
\end{abstract}

PALABRAS CLAVE: fin de la historia; forma de vida; animalidad; humanidad; dialéctica; ética; snob.

\section{Back to the end of history: an ethical interpretation of the posthistorical condition in Alexandre Kojève}

ABSTRACT: The following work aims to analyze and interpret Alexandre Kojève's theory of the end of history. The context of this research is mainly based on the footnote Kojève added in the second edition (1968) of his book Introduction à la lecture de Hegel. This footnote has led to intense debates and important philosophical questions. In this footnote, the paradigm of Japanese snobbery is taken as an alternative to the final animalization of posthistorical man. In this work, specifically, the kojevian rectification about the end of history will be put in relation to Kojève's work as a whole, his discussions with authors of his time and the references to the philosophical tradition. All this in order to unravel the ethical implications of the survival of the human in the end of history and its relationship with contemporary postmetaphisical approaches.

KEY WORDS: end of history; form of life; animality; humanity; dialectic; ethics; snob.

\section{Presentación}

En el presente artículo, se ofrece una interpretación de la filosofía de Alexandre Kojève basada, principal aunque no únicamente, en la célebre nota a pie de página añadida en la segunda edición de la Introduction à la lecture de Hegel ${ }^{2}$. La obra de Kojève permite una gran variedad de ejercicios hermenéuticos de estilo. Entre sus comentadores y admiradores, es muy común encontrar declaraciones de perplejidad y misterio. ¿Qué quiso decir realmente Kojève? ¿Qué se propuso? ${ }^{3}$ Nadie lo sabe

1 Este artículo es resultado del proyecto de investigación FFI2015-64858-P, titulado: Justicia y democracia: hacia un nuevo modelo de solidaridad, y financiado por el Ministerio de Economía y Competitividad (España)

2 KoJève, Introduction à la lecture de Hegel, 1968, pp. 436-437.

3 Dos ejemplos bastarán para mostrar esta perplejidad ante la filosofía de Kojève. El primero es de Allan Вцоом: «Podría uno preguntarse si Kojève no se encuentra realmente situado en algún punto entre Hegel y Heidegger, pero debemos agregar que el propio Kojève 
con certeza. Sin embargo, hay dos líneas tremendamente fecundas para trazar un plano interpretativo. La primera es, como es obvio, la cuestión de la historia y, en concreto, de la filosofía de la historia hegeliana. La segunda refiere a la cuestión ético-política, que a su vez podemos subdividir entre un nivel propiamente ético y un nivel propiamente político. Cruzando estas dos líneas, aparecen los grandes temas kojevianos: la filosofía del derecho, su concepción de la espiritualidad, la propuesta de una historia de la filosofía antigua (como parte de un proyecto inacabado de Système du Savoir), el debate sobre la secularización junto con el valor histórico del cristianismo, la cuestión de la tiranía y, por último, el mestizaje de las filosofías de Marx, por un lado, y Heidegger, por el otro, como síntesis de las dos polaridades que a su juicio marcarían la Filosofía contemporánea. Todo en Kojève es excesivo y muy poco académico. Aun así, para clarificar algunos de estos temas, el objetivo de estas páginas es considerar la dimensión ética de la filosofía kojeviana en el llamado "fin de la historia». Todo ello se referirá al ya mencionado pasaje clave de la nota a pie de página de la segunda edición de la publicación por parte de Raymond Queneau de los apuntes del famoso seminario dictado a l'École pratique des hautes études de París sobre la Fenomenología del espíritu de Hegel (bajo el título «La Philosophie Religieuse de Hegel»).

El célebre seminario de Kojève tuvo lugar entre los años 1933 y 1939. La primera edición del libro corresponde al año 1947. La segunda edición es de 1968, año, por cierto, de la muerte de Alexandre Kojève. Esta nota a pie de página es clave en más de un sentido. Se podría calificar, junto con su voluminoso Essai d'une histoire raisonée de la philosophie païenne, de testamento filosófico. Al fin y al cabo, en esta nota se revisa uno de los puntos fundamentales del paradigma kojeviano del fin de la historia: la animalización del hombre en el estado posthistórico ( «la fin de l'évolution historique de l'homme»). De hecho, este es el nudo de la argumentación de Kojève sobre el problema del Hombre, en mayúscula. En sentido estricto, solo puede existir el Hombre a lo largo de la Historia (también en mayúscula). El Hombre es un producto histórico (y dialectico, cabría añadir): él hace la historia y la historia lo hace a él. Es, pues, productor y producto de sí mismo. El Estado universal y homogéneo (que posibilita el reconocimiento recíproco con el que se concluye la negación histórica y activa en la lucha y el trabajo) es concebido bajo el paradigma de una obra -de ahí la pertinencia de caracterizaciones como «productor» y "producto». Pero realizada la obra, su autor ya no puede seguir actuando (construyéndola), con lo cual su capacidad eminentemente humana

lleva al lector a hacerse esta pregunta, la cual constituye un tema apropiado de reflexión filosófica. Kojève describe el carácter del saber aunque no da prueba de que éste haya sido realizado» (Bцoom, Gigantes y enanos, 1991, p. 261). El segundo, de Stanley Rosen: «The fundamental in Kojeve's teaching, which is in the deepest sense an attempt to explain himself to himself, is that it could not allow him to decide whether he was an administrator of automata, or a potential Japanese snob. As I believe he himself understood, his own discourse, with all its brilliance, came very close to silence.» (Rosen, Hermeneutics as Politics, 1987, p. 106)

4 Asistieron, entre otros, Raymond Aron, Maurice Merleau-Ponty, Éric Weil, Georges Bataille, Jacques Lacan, Raymond Queneau, Gaston Fessard, André Breton, Robert Marjolin, Roger Caillois, Denyse Harari y Jean Hyppolite. (Auffret, Alexandre Kojève. La philosophie, l'État, la fin de l'Histoire, 1990, pp. 238-239) 
(a través de la negación de lo dado, «negatividad-negadora») se vuelve espuria e insignificante, es decir, desaparece. Esta es la razón por la cual Kojève, en la primera nota a pie de página de la edición de 1947, afirmaba que

«En fait, la fin du temps humain ou de l'Histoire, c'est-à-dire l'anéantissement définitif de l'Homme proprement dit ou de l'Individu libre et historique, signifie tout simplement la cessation de l'Action au sens fort du terme. Ce qui veut dire pratiquement: - la disparition des guerres et des révolutions sanglantes. Et encore la disparition de la Philosophie; car l'Homme ne changeant plus essentiellement lui-même, il n'y a plus de changer les principes (vrais) qui sont à la base de la connaissance du Monde et de soi. Mais tout le reste peut se maintenir indéfiniment; l'art, l'amour, le jeu, etc., etc.; bref, tout ce qui rend l'homme heureux.» ${ }^{5}$

En cambio, la perspectiva ha cambiado parcialmente (pero no totalmente) en el añadido de 1968. Allí se señala que, en efecto, el fin de la Historia es algo que ya ha tenido lugar. Refiriéndose a la nota del año 1946, Kojève apunta que «le retour de l'Homme à l'animalité ne me paraissait pas impensable en tant que perspective d'avenir (plus o moins proche). ${ }^{6}$ Con todo, en este punto la situación todavía era la siguiente: ni se creía haber llegado realmente al fin de la Historia ni se podía decir que había una supervivencia (póstuma), más allá de la Historia, de lo humano (lo que habría sería reducción a la animalidad, adecuación a lo dado y mera adaptación a la naturaleza). La nota de la segunda edición implica una modificación de estas posiciones. Se podría presentar la secuencia de las posiciones kojevianas a partir del siguiente esquema cronológico:

- 1946: Kojève profetiza el retorno más o menos próximo a la animalidad.

- 1948: Kojève afirma que el fin de la Historia no solo ya está presente, sino que Hegel tenía razón en ver en la batalla de Jena el fin de la Historia. Las dos guerras mundiales son interpretadas como una extensión de los principios revolucionarios (alineación de las posiciones periféricas a la vanguardia de la Humanidad).

- 1948-1958: Constatación de que la forma «humana-animal» del fin de la Historia es el «American way of life».

- 1959: Cambio de tesis. A raíz de un viaje a Japón, revisa la conclusión de 19481958, con lo que ahora sostiene que en el fin de la Historia se mantiene una forma de vida "humana», cuyo paradigma es el esnobismo japonés ${ }^{7}$.

En Lo abierto. El hombre y el animal, Agamben sugiere que la segunda nota de Kojève «enuncia una serie de tesis sobre el final de la historia y sobre el estado presente del mundo, en las que no es posible distinguir entre la absoluta seriedad y una ironía igualmente absoluta $»^{8}$. Moverse entre estos dos absolutos, es fundamental para intentar esclarecer los objetivos y claves de la enigmática teoría de Kojève. El cumplimiento de la historia humana — que aparentemente debería conllevar la realización de todas las potencialidades humanas, su perfeccionamiento, y que como tal, en una perspectiva «hegelo-marxista», tiene un tono épico y de solemne

\footnotetext{
KoJÈve, Introduction à la lecture de Hegel, 1968, p. 435.

KoJÈve, Introduction à la lecture de Hegel, 1968, p. 436. 149-150.

Esquirol, "Alexandre Kojève, o com es distreu un hegelià al segle XX», 2006, pp.

8 Agamben, Lo abierto. El hombre y el animal, 2006, p. 24.
} 
gravedad (baste recordar solamente la presentación de la utopía comunista en Marx) - se convierte en una descripción cruda y descarnada de la sociedad de consumo (American way of life) en un tenor burlesco. De ahí, la «ironía absoluta». El referente escogido de la «sociedad sin clases» posthistórica es precisamente el líder hegemónico y representante par excellence del mundo capitalista (los Estados Unidos); y por otro lado, «los rusos (soviéticos) y los chinos (maoístas) no son más que americanos todavía pobres y, por otra parte, en vías de rápido enriquecimiento.» Que los Estados Unidos hayan alcanzado «el estadio final del comunismo marxista» es sin duda la tesis más sorprendente e increíble de esta figuración, es decir, constituye el zénit de la ironía kojeviana. Si algo brilla por su ausencia en la presentación posthistórica, es todo atisbo de crítica y negatividad en un «eterno presente» de la humanidad acabada. El final del tiempo humano se revela como una broma ácida y macabra.

\section{LA LUCHA POR EL RECONOCIMIENTO}

Nos hallamos en el terreno de la antropología filosófica de matriz hegeliana. Se trata de remontarse a la traducción comentada de la sección A del capítulo IV de la Fenomenología del Espíritu de Hegel, aparecida en Mesures (14 de enero de 1939), que conforma la obertura de la Introduction à la lecture de Hegel de nuestro autor. Conciencia, lucha, trabajo y reconocimiento son las notas que componen el acorde fundamental de esta filosofía que responde, con atrevimiento, a la cuarta pregunta kantiana: ¿Qué es el Hombre?

La respuesta es que el hombre es fundamentalmente Deseo; o dicho a la manera existencialista, el hombre no es, deviene. Comparte con el resto de los animales, el deseo de apropiarse de lo dado (en la alimentación, por ejemplo). Pero esto aún no es lo propiamente humano. Lo propiamente humano acontece con la lucha por el reconocimiento (el deseo deseado), que consiste en un plano que trasciende lo puramente animal. La animalidad, por sí misma, es insuficiente:

«L'être même de l'homme, l'être conscient de soi, implique et présuppose le Désir. Par conséquent, la réalité humaine ne peut se constituer et se maintenir qu'à l'intérieur d'une réalité biologique, d'une vie animale. Mais si le Désir animale est une condition nécessaire de la Conscience de soi, il n'est pas la condition suffisante. À lui seul, ce Désir ne constitue que le Sentiment de soi.»9

La oposición entre Sentiment y Conscience opera como fisura entre lo animal y lo humano (o mejor dicho, entre lo solamente animal y lo humano [más allá pero también] en lo animal). Pues bien, el advenimiento de lo humano es fruto del deseo del deseo del otro (esto es, del deseo de reconocimiento). Lo animal en lo humano es el soporte (animal antropóforo), a partir del cual (pero siempre fuera de él y por lo tanto no reductible a él) tiene lugar la antropogénesis o Historia humana ${ }^{10}$. El lector

9 KoJève, Introduction à la lecture de Hegel, 1968, p. 11.

10 La ontología de Kojève es dualista, entre otras razones, por su rechazo a la filosofía de la naturaleza hegeliana. «Le raisonnement de Hegel est certainement correct: si la Totalité réelle implique l'Homme, et si l'Homme est dialectique, la Totalité l'est aussi. Mais, en 
familiarizado con la Introduction à la lecture de Hegel de Kojève conocerá el fondo de la cuestión que aquí solo puede ser sumariamente esbozado. Lo nuclear es que los deseos de deseos implican la presencia del Otro. Para que exista la Conscience de soi, es absolutamente necesaria la «relación social» (aunque evidentemente esto no es así al modo clásico aristotélico de la sociabilidad natural humana). De hecho, el esquema hegelo-kojeviano rompe con la Identidad de lo natural, con lo dado. La Conscience de soi no es un atributo del animal humano ni una caracterización de la naturaleza humana. Objetos perfectamente inútiles desde el punto de vista biológico (una condecoración o la bandera del enemigo) son investidos de un brillo especial por el hecho de que son deseados por otros -y por consiguiente, son deseados en la medida en que son deseados por otros (prefiguración privilegiada del «deseo mimético» en Girard ${ }^{11}$ ). Es así como finalmente la historia humana se convierte en la historia de los deseos deseados, que constituye, a diferencia del deseo de algo real, el deseo de algo ideal. La noción de reconocimiento que opera en este contexto tiene connotaciones de «emulación» (Kojève), «honor» (Hobbes) y "gloria» (Maquiavelo). La acción en concordancia con este deseo de reconocimiento configura el mundo humano, desgajado del sistema instintivo (estímulo-respuesta) propio del reino animal.

El paso más allá de la animalidad es consecuencia de un acto de lucha por el reconocimiento. Lo decisivo de esta lucha es que supera el deseo animal por antonomasia (el deseo de autoconservación). Es evidente que, en esta tesitura, aparece un valor superior al valor de la mera supervivencia, con lo cual la formación de lo humano (superación del animal) implica tres aspectos complementarios que se integran en un mismo movimiento: a) una distinción jerárquica entre dos ámbitos de valor (lo deseado en términos del Sentiment de soi es subordinado a lo deseado en términos de lo que después será la Conscience de soi); b) una negación (negatividad-negadora) de lo dado animal en el hombre; c) una transcendencia respecto de lo animal en el hombre en función del deseo de reconocimiento. Siempre en un escenario social, estos tres aspectos se activan en forma de lucha: es la lucha a muerte por el reconocimiento (Hegel). Y esta es la génesis de la humanidad encuadrada en la hipótesis paradigmática del choque entre consciencias en la profundidad de una franja de la ultrahistoria. Este deseo de reconocimiento, como se ha dicho, sobrepasa lo dado «real» para alcanzar algún tipo de «ideal». La búsqueda de este «ideal» (de algo no natural) constituye la fuente de la Historia en sentido kojeviano («L'être vrai de l'Homme est son action»).

La lucha por el reconocimiento (basada en la igualdad de los contendientes) da como resultado una situación marcada por la diferencia (basada en la más absoluta desigualdad). El vencido debe sobrevivir y, para ello, es escrupulosamente necesario que no lleve hasta las últimas consecuencias el riesgo de su propia vida. Al final, debe agarrarse a ella y el vencedor, respetándosela, será aquel que habrá

partant de là, Hegel commet, à mon avis, une erreur grave. De la dialecticité de la Totalité réelle, il conclut à la dialecticité de ses deux éléments constitutifs fondamentaux, qui sont la Nature et l'Homme (= Histoire). (...) Il semble, d'ailleurs, qu'un dualisme ontologique soit indispensable à l'explication même de l'Histoire.» (KoJève, Introduction à la lecture de Hegel, 1968, pp. 485-486)

11 GiRARD, R. (1982). Le bouc émissaire. Paris: Grasset. 
conseguido exponer su propia vida para alcanzar el objetivo del reconocimiento. Uno reconoce su propia derrota (y reconoce al vencedor sin ser a su vez reconocido). $\mathrm{El}$ otro reconoce su victoria (y es reconocido sin a su vez reconocer al perdedor). Uno es esclavo y el otro amo. El Hombre nunca aparece como tal en la historia, sino siempre o bien como amo o bien como esclavo. No hay nada que $a b$ initio permita captar la figura del Hombre sin estos ropajes históricos que constituyen su aparición en el mundo. La antropología filosófica de Kojève está lejos de postular una esencia humana, sino que detecta con sagacidad las relaciones de dominación que forman el cuadro de la Historia humana. El humanismo no es una premisa, sino en todo caso un resultado proyectado al final de la Historia, una meta a alcanzar cuyo criterio no es transcendente (Strauss), sino históricamente inmanente.

«Le Maître est la Conscience existant pour soi. Et il n'est non plus seulement le concept [abstrait] de la Conscience mais une Conscience [réelle] existant pour soi, qui est médiatisée ave elle-même par une autre Conscience. À savoir, par une conscience telle qu'il appartient à sa réalité-essentielle d'être synthétisée avec l'être donné, c'est à dire avec la chosité en-tant-que-telle.» ${ }^{12}$

La revelación de la realidad humana no pasa solo por la lucha, sino también por el trabajo: definido como la tarea del esclavo cuya vida está en función del amo. Lo que a cambio de ver respetada su vida tiene que dar el esclavo al amo es precisamente el producto de su trabajo. Y la obra de la Historia humana, que parte de la lucha, se desarrolla a través del trabajo del esclavo, siendo el amo un paso necesario pero infértil en el desarrollo antropogénico (sans issue). Y evidentemente, el esclavo, al trabajar, también se separa del animal en la medida en que tiene que refrenar sus instintos (se forma y conforma). El esclavo, en definitiva, se forma como humano formando al mismo tiempo el mundo histórico como tal: su propia obra.

«Quand la Nature se transforme-t-elle en Welt (monde historique)? Quand il y a lutte, c'est-à-dire risque voulu de mort, apparition de la Négativité, qui se réalise en tant que travail. L'Histoire est l'histoire des luttes sanglantes par la reconnaissance (guerres, révolutions) et des travaux qui transforment la Nature. ${ }^{13}$

La historia humana (Histoire) es objetivada en el plano de la lucha por el reconocimiento, pero durante su transcurso tiene siempre la forma de un deberser. La cuestión fundamental es que el esclavo (ciudadano en potencia) tiende a actualizarse (la Revolución francesa, Robespierre-Napoleón, la batalla de Jena o incluso Stalin ${ }^{14}$ son referencias que Kojève emplea en distintos momentos para marcar este final). Y cuando lo hace, cuando la potencialidad se actualiza, entonces el resultado y fin de la Historia no permiten la supervivencia de un deber-ser que

12 KoJÈve, Introduction à la lecture de Hegel, 1968, p. 22

13 KoJÈve, Introduction à la lecture de Hegel, 1968, p. 55.

14 Raymond Aron explica en sus memorias que Kojève se declaraba «estalinista de estricta observancia» en los años treinta. Pero admite no entender en qué sentido lo decía, puesto que por otra parte Kojève en privado no tenía reparos en criticar el «barbarismo» de la URSS (ARon, Memoires: 50 ans de réflexion politique, 1983, p. 99). La lectura de la correspondencia de Kojève con Leo Strauss y su artículo «Tyrannie et Sagesse» podrían proporcionar algunas claves en este sentido. Por lo demás, Alexandre Kojève fue espía soviético durante treinta años. 
vaya más allá de lo dado (no hay negación de lo dado en el presente en función de un proyecto revolucionario de futuro). El Hombre, en calidad de ciudadano, alcanza la satisfacción (Befriedigung) al ser universalmente reconocido en su singularidad. Ya no se halla escindido entre amo y esclavo, sino que aparece de forma unitaria como Hombre en términos de una síntesis dialéctica entre estas dos figuras. Ello sucede solo al final, obviamente. El reconocimiento recíproco de los ciudadanos (ni amos ni esclavos) constituye la obra histórica del Estado universal y homogéneo (estacionario y sin forma, imposible de cambiar, destino último). La cuestión fundamental es ahora tratar de ver cuál es el lugar de lo humano en esta situación posthistórica. Y por ello, debemos volver a la nota añadida por Kojève en la segunda edición de la Introduction à la lecture de Hegel.

\section{2. ¿CiUdADANÍA O ANIMALIDAD?}

«Cet Homme est le citoyen de l'État parfait, qui est pleinement et définitivement satisfait par cet État universel et homogène. Rien ne change donc plus et ne peut plus changer dans cet État universel et homogène. Il n'y a plus d'Histoire, l'avenir y est un passé qui a déjà été, la vie y est donc purement biologique. Il n'y a donc plus d'Homme proprement dit. $\rangle^{15}$

La paradoja del ciudadano en sentido estricto (distinto del ciudadano del estado antiguo, por ejemplo) es que se ha vuelto conforme con la realidad, esto es, no la niega; es por ello que se puede decir que se adapta a su entorno al modo del animal. Evidentemente, este esquema rompe tajantemente con la oposición clásica entre la vida natural (zoé) y la vida cualificada cívicamente (bíos). Esta distinción entre las dos formas de vida — que Foucault ${ }^{16}$ presenta en sus cursos al Collège de France y en el último capítulo de su primer volumen de la Histoire de la sexualité (La Volonté de savoir) — es el modo tradicional de presentar la vida del ciudadano o vida cívica en contraposición con la mera vida natural. Según Aristóteles, las formas de vida están limitadas en el círculo de la polis. En el esquema hegeliano, en cambio, lo análogo al bíos se construiría históricamente (en la interpretación kojeviana, mediante las figuras de la fenomenología, tales como el estoicismo, el escepticismo o el cristianismo). Lo que ocurre es que al término de la Historia las posibilidades del bíos vuelven a ser limitadas históricamente, en una circularidad que el Sabio contemplaría ${ }^{17}$. Pues bien, en Kojève, finalmente, el bíos cívico se

15 KoJève, Introduction à la lecture de Hegel, 1968, p. 387.

16 Foucault, M. (2014). Subjectivité et vérité. Cours au Collège de France. 1980-1981, Paris: Gallimard-Seuil, pp. 36-37 y 253-256; cfr. Id., Foucault, M. (2006). La hermenéutica del sujeto (Curso en el Cóllege de France 1981-1982), Buenos Aires: Fondo de Cultura Económica, p. 464, nota al pie 28 .

17 Las «formas de vida» en Aristóteles serían limitadas en número: se podrían representar en un círculo fundamentado teleológicamente y en el escenario de la polis, en cuyo centro irradiador estaría el bíos theoretikós. Las «formas de vida» en Hegel (siempre según Kojève) también serían limitadas en número: pero el círculo en el que se podrían representar estaría fundamentado dialécticamente y en el escenario de la Historia, y cuyo centro estaría reservado a la Sagesse. 
convierte en zoé humana (en términos hegelianos, se podría hablar perfectamente de síntesis o reconciliación entre las dos instancias). En esta obra (que es el Estado universal y homogéneo), el Hombre se reconcilia con su animalidad («la vie y est donc purement biologique»), con lo cual Kojève puede afirmar en la nota que los animales de la especie Homo sapiens «réagiraient par des reflexes conditionnés à des signaux sonores ou mimiques et leurs soi disant "discours" seraient ainsi semblables au prétendu "langage" des abeilles.» ${ }^{18} \mathrm{El}$ panorama es truculento si se lo complementa con la especificación que Kojève presenta en una carta a Leo Strauss del 19 de septiembre de 1950:

"Besides, "not human" can mean "animal” (or, better -automaton) as well as "God." In the final state there naturally are no more "human beings" in our sense of an historical human being. The "healthy" automata are "satisfied" (sports, art, eroticism, etc.) and the "sick" ones get locked up. As for those who are not satisfied with their "purposeless activity" (art, etc.), they are philosophers (who can attain wisdom if they "contemplate" enough). By doing so, they become "gods". The tyrant becomes an administrator, a cog in the "machine" fashioned by automata for automata. ${ }^{19}$

El cruce de ambos textos es fundamental para entender esta posición como una paráfrasis kojeviana del clásico paso aristotélico que opone la vida en la polis (bíos), tanto al dios como al animal (aquel que «no es parte de una ciudad, sino una bestia o un dios» [Política, 1253a14]). Si mantuviéramos el modelo de Aristóteles, tendríamos que decir que la sociedad del estado final («estado» en el sentido político pero también en el sentido «estático» y «presente», como «lo que está») no es otra cosa que Naturaleza (physis) que desagrega las relaciones sociales y aísla los individuos en su universalidad bajo la categoría solitaria del animal (autómata) o la del dios (Sabio). Pero lo realmente interesante en esta cuestión de la máxima importancia es que el final state reconcilia la oposición entre nomos y physis. La obra del Hombre — su acción histórica, lucha y trabajo mediante- supone una civilización de la naturaleza y una naturalización de la civilidad. El cociente de la operación es equivalente a la animalización (el ciudadano deviene autómata, como los animales-máquina de Descartes). O bien pasa a la categoría de dios (el Sabio), como aquel que dispone de la Sagesse que permite comprender todo lo dado históricamente, sin que cualquier otro episodio o figura fenomenológica pueda convertirse en un punto que lo comprenda a él. «D’une manière générale, un homme (individuel ou collectif) est supérieur aux autres dans la mesure où il les comprend, sans pouvoir être compris par eux. ${ }^{20} \mathrm{La}$ "aparente inactividad» del Sabio consiste en comprender hegelianamente (tout comprendre, c'est tout pardonner) el desarrollo histórico que ha posibilitado la realización del final state: reconciliación automática del hombre con su naturaleza, vida adaptada a la machine (o sistema) y lenguaje no discursivo-filosófico (puramente comunicativo al modo de las aristotélicas abejas $\left.^{21}\right)$, carente de Palabra (logos).

18 KoJÈve, Introduction à la lecture de Hegel, 1968, p. 436.

19 Strauss, On Tyranny. Revised and Expanded Edition. Including the Strauss-Kojève Correspondence 2000, p. 255.

20 KoJÈve, Introduction à la lecture de Hegel, 1968, p. 404.

${ }^{21}$ Metafísica, A, 980b 
La política a lo largo del tiempo (que es «herencia» de la polis ${ }^{22}$ ) no ha sido otra cosa que el puente o mediación necesaria para cerrar la fisura entre el mundo histórico y la naturaleza. El Hombre (histórico) era un producto-productor de su mundo (en las tentativas de acción y, sobre todo, en el error). El final state anula el error histórico (ideologías, revoluciones, guerras, etc.) y lleva a le vrai (lo verdadero); pero con esta anulación, solo nos quedamos con los despojos del Hombre (el Homo sapiens como especie en un entorno de "reflejos condicionados», «engranajes en una máquina» o «autómatas»). La satisfacción del Deseo de reconocimiento se plasma en una indistinción entre zoé y bios — «la Nature étant définitivement domptée, c'est-à-dire harmonisée avec l'Homme.» ${ }^{23}$ Es muy significativo que este fragmento citado se encuentre en la primera nota a pie de página, revisada por la de la segunda edición, cuyo contexto es el paso marxista del "Reino de la necesidad» (Reich der Notwendigkeit) al Reino de la libertad (Reich der Freiheit) ${ }^{24}$. Pero la libertad, en la administración de las cosas (en la postpolítica), como se pregunta Carl Schmitt en su Begriff des Politischen, ¿es libertad para qué? La libertad, esencialmente, ha sido la condición por la que el Hombre se construía su propia obra. Entonces era libre. Kojève constata que esta libertad histórica es sustituida por la felicidad (propia de la sociedad sin clases de la utopía comunista en la que solo queda «tout ce qui rend l'Homme heureux»). Inusitada permuta de la libertad por la felicidad en el fin de la historia.

En este sentido, la obra humana (evidentemente, merced a la técnica) se ha convertido en el hábitat de los descendientes del Hombre histórico. La visión de este mundo invierte la utopía en una distopía inquietante donde sus habitantes son reducidos a la condición de animal-autómata «sano» (healthy). También está el «enfermo» (sick), por supuesto. Los inadaptados, por el contrario, tendrán que ser encerrados (locked up) en manicomios o prisiones. Por último quedan otro tipo de insatisfechos (sanos insatisfechos), que podrán dedicarse a la Filosofía con la esperanza de devenir Sabios (en sentido hegeliano, esto es, persuadidos racionalmente de la justificación del desarrollo de un proceso histórico, y por lo tanto reconciliados con el Todo [Verdad es Totalidad]). El panorama resultante es una variación hegeliana del tema de la «jaula de hierro» de Weber —animales domesticados (domptés) en la jaula de la administración propia del Estado universal y homogéneo, engranajes en la cadena. Los demás, los insatisfechos, deberán aprender a filosofar apostando, a lo más, por repetir a Hegel.

\section{LA RECTIFICACIÓN: ESNOBISMO POSTHISTÓRICO}

La nota de la segunda edición de la Introduction à la lecture de Hegel está contundentemente condensada. Son aproximadamente dos páginas que cambian el sentido de la conclusión kojeviana del libro. Como es habitual en nuestro autor, se deja al lector la tarea de pensar y concretar lo que ahí se anuncia concisa y

22 AREndt, Men in Dark Times, 1968, p. 206.

23 KoJÈve, Introduction à la lecture de Hegel, 1968, p. 435.

24 MARx, K.: El Capital, libro III, cap. 48. 
crípticamente. Nada se sirve masticado. No obstante, la base de la teoría kojeviana es explícita: el fin de la historia se da por hecho desde la batalla de Jena (1806), con lo cual el tono profético de una Revolución a venir desaparece.

«Du point de vue authentiquement historique, les deux guerres mondiales avec leur cortège de petites et grandes révolutions n'ont eu pour effet que d'aligner sur les positions historiques européennes (réelles ou virtuelles) les plus avancées, les civilisations retardataires des provinces périphériques. $\rangle^{25}$

Todo: las dos guerras mundiales, el nazismo (truchement de l'hitlérisme), el socialismo real (soviétisation de la Russie et communisation de la Chine) y los procesos de autodeterminación del Tercer Mundo (Togo, Papúa) es encuadrado en lo que podríamos llamar un «esquema imperial». La semilla incipiente del nuevo orden mundial ya ha germinado, pero todavía no ha dado todos sus frutos. Es una cuestión puramente pragmática o técnica (expansión en el espacio) el hecho de que finalmente todos los confines del Imperio lleguen al estadio del American way of life. "D'ores et déjà, ce processus d'élimination (des séquelles plus ou moins anachroniques) est d'ailleurs plus avancé dans les prolongements nordaméricains de l'Europe qu'en Europe elle même. ${ }^{26}$ Alineación en el estadio final del «comunismo marxista» concretado en la América fordista ${ }^{27}$. Europa como espacio aún retardatario en contraposición con las viejas tesis kojevianas del Empire latin de 1945, donde el Mediterráneo era presentado como la vanguardia de la humanidad. Animalización como equivalente a consumismo, siendo el actual neuromarketing, por ejemplo, un perfecto exponente de la concepción del individuo como autómata que hubiera hecho las delicias de un Kojève ávido de ejemplificaciones. Pero aún hay más: aquí el Hombre ya no puede ser calificado de feliz (en los términos de una ética eudemonista), sino solamente de "satisfecho» o "contento», en la medida en que se «contentará» con lo dado.

Kojève rectifica: unos viajes a Japón (1959) le han convencido de la necesidad de cambiar «d'avis sur ce point». Será fundamental demorarse en este punto porque aquí es donde aparece no tanto una proyección geopolítica harto anecdótica e inverosímil (niponización del mundo contra americanización del mundo), como una reflexión de carácter ético que debe ser tratada con sumo cuidado: la problemática de la negación formal (esnobismo) en el fin de la historia. Vale la pena citar íntegramente este famoso párrafo:

«La civilisation japonaise "post-historique” s'est engagée dans des voies diamétralement opposées à la "voie américaine”. Sans doute, n’y a-t-il plus eu au Japon de Religion, de Morale, ni de Politique au sens "européen" ou "historique" de ces mots. Mais le Snobisme à l'état pur y créa des disciplines négatrices du donné "naturel" ou "animal" qui dépassèrent de loin en efficacité, celles qui naissaient, au Japon ou ailleurs, de l'Action "historique", c'est-à-dire des Luttes guerrières et révolutionnaires ou du Travail forcé. Certes, les sommets (nulle part égalés)

25 KoJÈve, Introduction à la lecture de Hegel, 1968, p. 436.

26 KoJÈve, Introduction à la lecture de Hegel, 1968, p. 436.

27 KoJÈve, A. (1980/1). «Capitalisme et Socialisme. Marx est Dieu, Ford est son prohète», Commentaire, n. 9, pp. 135-137. Ver también: De VRIEs, E. (2001). "Alexandre Kojève - Carl Schmitt Correspondence and Alexandre Kojève, "Colonialism from a European Perspective», Interpretation, 29/1, pp. 91-130. 
du snobisme spécifiquement japonais qui sont le Théâtre Nô, la cérémonie du thé et l'art des bouquets de fleurs furent et restent encore l'apanage exclusif des gens nobles et riches. Mais, en dépit des inégalités économiques et sociales persistantes, tous les Japonais sans exception sont actuellement en état de vivre en fonction de valeurs totalement formalisées, c'est-à-dire complètement vidées de tout contenu "humain" au sens d'"historique". Ainsi, à la limite, tout Japonais est en principe capable de procéder, par pur snobisme, à un suicide parfaitement "gratuit" (la classique épée du samouraï pouvant être remplacée par un avion ou une torpille), qui n'a rien à voir avec le risque de la vie dans une Lutte menée en fonction de valeurs "historiques" à contenu social ou politique. Ce qui semble permettre de croire que l'interaction récemment amorcée entre le Japon et le monde occidental aboutira en fin de compte non pas à une rebarbarisation des Japonais, mais à une "japonisation" des Occidentaux (les russes y compris). ${ }^{28}$

$\mathrm{Y}$ es que «ningún animal puede ser snob». Así pues, las formas se desprenden de los contenidos y el «Sujeto se opone al Objeto» igualmente (como ocurría en la Historia), pero no para transformarlo en sentido histórico, sino simplemente para oponerse a sí mismo y a los otros como una forma a cualquier contenido dado. Se podrá hablar, por consiguiente, de una negación puramente formal, gratuita y autotélica en el período posthistórico — de una negación que ya no conlleva síntesis o reconciliación posible (no metafísica). Esta es la supervivencia de lo humano en el animal que le sirve de soporte (animal antropóforo). Esclarecer el significado de esta posición es fundamental. De hecho, la referencia a Japón, por espectacular, puede dar lugar a equívocos y desconciertos. Ciertamente, el paradigma nipón de lo snob funciona aquí como "tipo ideal» en sentido weberiano (o como "paradigma» en sentido agambeniano) de la actividad humana más allá de la obra (fruto de la Acción histórica de Lucha y Trabajo) realizada. Esta obra es el hábitat humano en el final state (el Estado universal y homogéneo). La casa común es el mundo civilizado - la decoración y el diseño de interiores, por decirlo así, es todo lo que resta en forma de gestión y administración de lo dado. Pero lo snob, justamente, indica el tipo de actividad cultural que bloquea la animalización definitiva del ser humano. El autómata de antaño es remplazado por la práctica (praxis) del esnobismo: estilo de vida antes monopolio del noble, pero actualmente, siendo kojevianamente optimistas, en proceso de democratización progresiva.

La historia de la ética y la filosofía política nos sirven de apoyo y acuden a nuestra ayuda para comprender esta rectificación de Kojève en toda su complejidad. La nota es breve, pero contiene un alto grado de densidad. Se parte de la satisfacción de las necesidades materiales (abundancia y seguridad) y del deseo de reconocimiento (reconocimiento recíproco en la ciudadanía universal). Ahora bien, en este punto ya no se trata de concluir con un retorno al animal, sino de mantener el proceso de negación en lo que podríamos llamar «actividad» para distinguirlo de la «acción». La actividad es un movimiento circular de perfeccionamiento que no tiene fin más allá de su propio ejercicio; la acción, en cambio, es proyección lineal enfocada a un resultado. Sin duda, el referente no explícito (más allá de la alusión a las costumbres japonesas) es precisamente la idea de ética (ethos) que encontramos en la tradición filosófica occidental, concretamente en la época clásica. Ethos en

28 KoJève, Introduction à la lecture de Hegel, 1968, p. 437. 
el sentido de costumbre, ciertamente, pero no de carácter — puesto que, como veremos, el carácter formaría parte de los contenidos que deben ser negados en las nuevas y perfeccionadas costumbres (formales). También en el caso de Aristóteles, la ética es ciertamente un privilegio aristocrático, un tesoro reservado a una minoría compuesta por ciudadanos, propietarios y varones, reservado, en suma, a una élite de nobles (perspectiva del amo). Se trataría de entender el esnobismo, literalmente, como un conjunto de normas de etiqueta y, en consecuencia, como puro esteticismo popularizado y accesible al ciudadano (ni amo ni esclavo o los dos a la vez).

«Kojève eventually came to slightly more hopeful view of the end of history, in which human negativity, though no longer a force for political change, might be preserved in a kind of aestheticism. The impetus for this third and final version of the end of history came not from a philosophical reading or reflection, but, he tells us, from a visit to Japan in 1959. ${ }^{29}$

F. Roger Devlin en su Alexandre Kojève and the Outcome of Modern Thought no presta demasiada atención al significado de la nota de la segunda edición de la Introduction à la lecture de Hegel. En su cómputo de versiones del final de la historia, de hecho, entiende que o el fin de la historia es una proyección de un futuro próximo (primera versión), o ya se ha dado en la batalla de Jena de 1806 y completado en el American way of life (segunda versión), o bien está encarnado en el «esnobismo japonés» (tercera versión). En cualquier caso, Devlin identifica el esnobismo como puro esteticismo. Ciertamente, hay un elemento evidente de esteticismo o culturalismo (de gratuidad artística) en esa concepción. Con todo, ello no es lo nuclear de la posición kojeviana. Devlin, por lo demás, va aún más lejos en su comentario:

"A civilization hardly influenced by Greece or by Christianity made an "experiment" in the end of history (that is, in the homogeneity, and with isolation providing an ersatz universality) two hundred years before Napoleon and Hegel! Taken literally, this remark liquidates the rest of Kojeve's teaching. For it implies that the distinguishing features of the Endstate are attainable or replicable apart from the unique series of free acts of work and struggle which make up European history. But this, of course, is just the traditional doctrine of natural right: that man's end is equally attainable (or unattainable) everywhere and at all times. Perhaps Kojeve's remarks are partly ironic. But then it is difficult to say just what the example of Japan does show us.» ${ }^{30}$

¿Qué nos muestra el ejemplo de Japón? ¿Se trata de una impugnación radical de todo el discurso kojeviano? La enigmática nota de la segunda edición, según Devlin, excede el planteamiento eurocéntrico de Kojève y muestra, por ejemplo, un Endstate no subordinado al proceso histórico de la Lucha y el Trabajo que pasa por Grecia (filosofía) y Jerusalén-Roma (cristianismo). Ello lo remite a la teoría opuesta al «modernismo» kojeviano: la teoría del derecho natural (iusnaturalismo). Pero la cuestión de fondo es que Devlin olvida que lo snob japonés está siendo interpretado deliberadamente desde la perspectiva histórica (hegeliana). Sin esta

29 Devlin, Alexandre Kojève and the Outcome of Modern World, 2004, p. 144.

30 Deviln, Alexandre Kojève and the Outcome of Modern World, 2004, pp. 145-146. 
clave hermenéutica, no tendría sentido establecer el paradigma del Japón reformado como fase última de la historia humana. Kojève busca referentes (American way of Life, Imperio socialista, esnobismo japonés) para ilustrar las implicaciones de una concepción antropológica y dialéctica de la Historia. Pero no se debe confundir lo señalado con la señal: todo paradigma no se basa en describir lo que es, sino en representarlo analógicamente (Melandri $\left.{ }^{31}\right)$.

Lo snob, además, también puede tener su lado sombrío (y no necesariamente «esteticista» y autocomplaciente). Bastará con leer unas consideraciones de Erik de Vries en su edición de la correspondencia entre Carl Schmitt y Alexandre Kojève a propósito de esta espinosa cuestión para cerciorarse de ello:

«According to this interpretation, posthistorical man's capacity for mortal risk is gratuitous and therefore apolitical. If posthistorical man's choice between animalization and gratuitous suicide is determined, as Kojève suggests in the Introduction, by his willingness to be satisfied by the recognition the universal and homogeneous state provides him, then the gratuitousness of the latter option lies in the impossibility to achieve recognition through action. The kamikaze pilot Kojève uses as an example in 1959 has been replaced in our time by the suicide bomber, and, perhaps, in the near future, a radical wing of anti-WTO protesters: the value for which he perishes (or risks perishing) is "formal" rather tan historical; that is, any success is temporary, and will be forgotten once overcome. Posthistorical men cannot negate the universal and homogeneous state because it is formless (see Schmitt's letter of Dec. 14, 1955, below) and will not play the enemy.» ${ }^{32}$

Y es que en la nota de la segunda edición de la Introduction à la lecture de Hegel Kojève también habla, literalmente, del riesgo mortal en el sentido del kamikaze (como descendiente del samurái), con lo cual el mundo contemporáneo, con el advenimiento del terrorismo global, nos brinda una cruenta actualización de esta actividad posthistórica. La perspectiva esteticista, en este sentido, es totalmente desbordada por un tipo de acción que se puede calificar de «apolítica» o directamente de nihilista ( sin contenido). El fin de la historia no es necesariamente pacífico; simplemente ocurre que la violencia es, en este estadio, completamente gratuita («dialécticamente inconvertible», en términos de Étienne Balibar ${ }^{33}$ ). De Vries aventura que una posible ala radical del movimiento altermundista podría perfectamente recrear el acto del suicido gratuito por una causa formal —al fin y al cabo, el Endstate no tiene forma (formless), con lo cual no es esencialmente modificable (otro mundo no es posible) a través de la lucha política (histórica). Así, toda intervención en el Estado universal y homogéneo es perfectamente factible sin el uso de la violencia, con lo cual el riesgo mortal posthistórico se revela a todas luces inane.

Para terminar en algún lugar con las interpretaciones de esta nota kojeviana, es importante añadir una precisión crucial sobre el posible sentido del esnobismo

31 Melandri, E. (2004). La linea e il circolo. Un studio lógico-filosofico sull'analogia. Macerata: Quaderni Quodlibet.

32 De VRIES, Alexandre Kojève - Carl Schmitt Correspondence and Alexandre Kojève, «Colonialism from a European Perspective», 2001, pp. 93-94.

33 Balibar, É. (2010). Violence et civilité. Wellek Library Lectures et autres essais de philosophie politique, París: Galilée. 
posthistórico. Esta vez el referente de interpretación es un texto inédito de Kojève de 1945, publicado póstumamente en el número 1 de La Règle du Jeu de BernardHenry Lévy:

«This notion of japanized snobbery is part of a coherent vision of how aristocratic sensibilities might balance the rule of the masses through the consolidation of the State power. In 1945, Kojève wrote a foreign policy memo for Charles de Gaulle designed to plot France's foreign policy for the postwar era. He outlines a «Latin» or «catholic» Empire that would provide a home for Latin civilization as a mediating bulwark between the Anglo-Saxon Protestant Empire and the SovietOrthodox Empire, in order to preserve and protect the values of the «humanization of free time» and introduce "aristocratic sweetness of living» into bourgeois well-being. The alternative to the barbaric Statism of Stalinism and the invidious self-interest of capitalism is found in the unity of France, Spain, and Italy, brought together by (1) their shared Catholicism, (2) their close language, and (3) and most importantly, a unit of civilization and what Kojève calls a Latin mentality. By preserving the political Independence of the Latin Empire, a world in which «Latin» values exist is created; the cultivation of the art of leisure through the transformation of bourgeois well-being into aristocratic sweetness of living, and «delight» in pleasure. This would justify itself "to the world and to history» ${ }^{34}$.

Así, el Imperio latino, en la medida en que posibilita el máximo de tiempo de ocio para todos sus ciudadanos, se convierte en paradigma y modelo del cosmopolitismo posthistórico. El catolicismo latino se contrapone al protestantismo (Weber) y al imperio ortodoxo de la Unión Soviética. Según Kojève, el Imperio anglosajón protestante encarna el principio del capitalismo: es la tesis weberiana del ascetismo mundano. La laboriosidad protestante sería inferior, a ojos de Kojève, al espíritu del catolicismo romano. Por otro lado, el estatismo bárbaro del imperio soviético ortodoxo tampoco es una alternativa en el texto de 1945. El imperio latino (católico) supone el ideal mediterráneo del ocio y el dolce far niente. Los valores latinos son el arte de cultivar el ocio y la dulzura de vivir, lo cual «se justifica a sí mismo, al mundo y a la historia.»

En realidad, se trata de ver la conexión entre Aristóteles y Marx, en el sentido de que el progreso se basa en asegurar el máximo de ocio para la humanidad. En Aristóteles, la gran masa de la población es obviamente excluida de este "progreso». En Marx, en cambio, se trata precisamente de universalizar las conquistas políticas en el socialismo: al decir de Hannah Arendt, el objetivo es «la polis sin esclavos» ${ }^{35}$. Pero lo que en principio sería el logro supremo de la humanidad se invierte en su contrario a través de la animalización del hombre en la sociedad de consumo (tesis de la alienación moderna del mundo en Arendt). Y aquí es donde el modelo kojeviano del esnobismo ofrece una particular alternativa al consumismo del American way of life fundamentado en la metabolización del hombre con la naturaleza a través del labour. El esnobismo introduce la cualidad en un mundo sin ideales, un mundo

34 Murphy, «Alexandre Kojève: Cosmpolitanism at the End of History», 2011, p. 203. También se defiende esta tesis en Howse, R. (2006). "Europe and the New World Order: Lessons from Alexandre Kojève's engagement with Schmitt's "Nomos der Erde" », en: Leiden Journal of International Law, 19, p. 9.

35 Arendt, The Human Condition, 1958, p. 133. 
consumado, un mundo sin mundo. Esta cualidad es el perfeccionamiento continuo propio de la vida ética en la polis griega (Aristóteles), pero esta vez universalizado en una sociedad mundial homogénea. Esto es, equivale a una salvaguarda del elemento aristocrático en su síntesis con el bienestar burgués y evita, precisamente, el embrutecimiento del resto humano en la posthistoria («ningún animal puede ser snob»). La exigencia de fondo es forzosamente ética: la «efectividad» (Kojève) de la lucha contra uno mismo que representa el desgaje de la forma humana sobre el contenido animal (material), y que presupone un ciclo infinito de negación (Lucha y Trabajo) con uno mismo y los demás. En esto consiste la supervivencia del Hombre más allá de la Revolución que concluye la Historia y, por esto mismo, el autómata condicionado por la machine es superado por una exigencia que radica en una nueva modalidad de Deseo (o «potencialidad débil»). A todos ellos, animales o snobs, no obstante, se sigue oponiendo la figura quieta del Sabio-dios que solo es posible cuando el Tiempo humano ha terminado. Él es el único que no cambia (en la pura contemplación de la Verdad circular ya realizada). Aunque también el logos acabe por desaparecer en el fin de la historia.

\section{LA CONCLUSIÓN: ETICISMO POSTHISTÓRICO}

El esnobismo se presenta como una «forma de vida» (bios) y no como mera vida animalizada (zoé). Este punto es clave, puesto que en realidad la rectificación de la nota de la segunda edición apunta a la conservación de una cualificación de la vida, a un deber de cualificación. Por mucho que los valores sean puramente formales (ejemplificados en la ceremonia del té o los arreglos florales japoneses), la resonancia del texto es ética. El puro esteticismo (o formalismo), dicho de otro modo, se justifica éticamente en la medida en que se trata precisamente de una «forma» de vida. Para concluir, en este apartado se señalaran los elementos que permiten una lectura ética de la nota de la segunda edición de la Introduction à la lecture de Hegel.

En primer lugar, el elemento ético tiene un anclaje histórico. «Snobbery becomes the psychological state of aidos or what is associated with the practices of the aristo $i$ or aristocracy.» ${ }^{36}$ Todo pasa como si la praxis marxista que ha cambiado el mundo volviese a su origen de praxis aristotélica en el fin de la historia. Como diría Kojève mismo: "All this seems to me rather "classical". With the one difference that according to Hegel this is not right from the start, but only becomes right at the end.» ${ }^{37}$ La Acción deviene «actividad» praxial sin fin, lo cual solo es posible con la separación de la forma sobre todo contenido posible, de ahí que

36 Darby, The Feast: Meditations on Politics and Time, 1990, pp. 174-176. Citado y comentado en Goldstein, J. D. (2006). Hegel's Idea of the Good Life. Dordrecht: Springer, p. 227-28, nota al pie 25. Goldstein sostiene que «snobbery replaces ethical habit in Japanized man». Sin embargo, admite que, aunque se pierda el Bien, la propuesta kojeviana apunta a una solución a la banalidad mediante la «arbitrariedad»(arbitrariness). ¿Pero no sería igualmente ocasión para una ética sin metafísica?

37 Strauss, On Tyranny. Revised and Expanded Edition. Including the Strauss-Kojève Correspondence, 2000, p. 255. 
se pueda calificar irónicamente de «formalismo» o «esnobismo». Ciertamente, cualquier ética de la historia (destinada a las élites o "grupo exclusivo» propio de cada sociedad histórica) nos parecería igualmente snob, como de hecho es «snob», según Kojève, la vida de las clases altas en el Japón de la reforma de Hideyoshi. La única diferencia es que en la pintura kojeviana el esnobismo se universalizaría con el advenimiento de la sociedad homogénea a escala mundial ${ }^{38}$. No es de extrañar que, en este contexto, una posible americanización de los japoneses sea vista bajo el prisma de una amenazante «rebarbarización», precisamente.

En segundo lugar, la negatividad que implica la acción débil en la posthistoria es una variación de la "negatividad sin empleo» del discípulo de Kojève Georges Bataille. Esto ha sido señalado más de una vez:

«What kind of Action can this be, and why Japanese snobbery, which for him is the main characteristic of Japanese culture, has made this weak Japanese end of History happen? The answer to these questions, we believe, must be sought, rather than in Japan itself, in Kojève's own thought, that is to say in his interpretation of Hegelian dialectics and in his close relationship with the thinker of Negativity with No Use, Georges Bataille.» ${ }^{39}$

Giorgio Agamben, por otro lado, constata que «el hecho de que el hombre sobreviva a la historia en la forma del snobismo japonés parece una versión más elegante (aunque, tal vez, paródica) de aquella "negatividad sin empleo» que Bataille trataba de definir a su modo, ciertamente más ingenuo y que a los ojos de Kojève debía parecer de mal gusto. ${ }^{40} \mathrm{El}$ snob, en este sentido, sería "soberano» en la concepción bataillana de la filosofía del instante ( «eterno presente» de Kojève), en el que la negación no se subordina a nada y se torna excesiva por sobreabundante (el gasto sin reserva de Bataille). Nuevamente, la nota kojeviana muestra un elemento ético dibujado en una forma de vida que rompe con el cómputo instrumental y burgués del tiempo (economía restringida) e ilumina, por ello, posibilidades liberadoras de una vida soberana (economía general). "Digamos que el soberano (o la vida soberana) comienza cuando, asegurado lo necesario, la posibilidad de la vida se abre sin límite.» ${ }^{41}$

En tercer lugar, ya Kojève había tratado el esquema del formalismo en su Esquisse d'une phénomenologie du droit, concretamente en sus reflexiones sobre la

38 ¿Se puede realmente universalizar el esnobismo? En un texto de 1942, Kojève escribía: «También está el fenómeno universalmente difundido, que se llama "esnobismo". El esnob es un hombre que se imagina ser "original", "personal”, etc., pero que de hecho es esclavo de una Autoridad (no menos que el "pequeño burgués"), del "qué dirán”; él solamente reconoce la Autoridad de lo que cree ser la "élite", suponiendo que esta última sea necesariamente una Minoría. Sentimos la tentación de decir que hay casos (el "esnobismo", por ejemplo) donde la Minoría ejerce una Autoridad sui generis por el solo hecho de ser una Minoría, de la misma manera que hay casos (el "hombre prudente", por ejemplo) en los que la Mayoría ejerce una Autoridad en tanto Mayoría. (...) La Autoridad que reviste una Minoría se "justifica" o se explica por la "cualidad" y no por la cantidad. (Hasta el "esnob" aduce pertenecer a una élite y no a la minoría.)» (KoJÈve, La noción de Autoridad, 2006, pp. 61-62)

39 Aingeru, «The Japanese End: Japan in Alexandre Kojeve's End of History», 2009, p. 65.

40 Agamben, Lo abierto. El hombre y el animal, 2006, p. 27.

41 Bataille, Lo que entiendo por soberanía, 1996, p. 64. 
moral. Uno de los modos en que la separación entre forma y contenido puede tener lugar es precisamente en la moral en sentido kojeviano. "Le Moraliste ne réalise la Morale que pour devenir (moralement) parfait lui-même (...) En tant que Moraliste il ne s'interesse qu'à sa propre perfection morale.» ${ }^{42}$ Pero, dado que la perfección moral es un ejercicio continuo, el moralista se convierte en un perpetuo snob que se mejora a sí mismo indefinidamente, sin necesidad de actuar para transformar el mundo en función de un ideal a realizar (como es la Justicia en la fenomenología del derecho). Ya no hay, empero, ninguna opción para una transformación radical del Hombre en el sentido histórico, puesto que incluso «le Moraliste parfait n'est que "moralement" supérieur aux autres, sans s'en distinguer radicalement.» ${ }^{43} \mathrm{El}$ ejercicio interior de la Moral en la Esquisse d'une phénomenologie du droit, por lo tanto, sería un exponente avant la lettre de este tipo de relación agonística con uno mismo que es propia del esnobismo posthistórico.

En cuarto lugar, finalmente, cabe considerar la dimensión temporal en el planteamiento posthistórico. En efecto, si, como dice Kojève, un Sujeto sigue oponiéndose a un Objeto, entonces esta negatividad débil, distinta de la negatividad histórica, lleva a pensar en una nueva forma de exigencia. "Pour rester humain, l'Homme doit rester "un Sujet opposé à l'Objet», même si disparaissent l'Action négatrice du donné et l'Erreur (...) et doit continuer à détacher les formes de leurs contenus." ${ }^{44}$ Kojève habla aquí de un deber (doit) enfocado a un porvenir que ya no es histórico ( «il faut l'avenir», como dice Derrida). "Revising and contesting his first hypothesis, Kojève comes around to thinking that there is an even more final end of history, an even more eschatological end than the American (and even Californian, as he says somewhere) "happy end". ${ }^{45}$ Ahora bien, el fin de la historia no implica un final cerrado, sino una forma de tiempo abierta, se trata del «tiempo del fin» (Agamben) más que del «fin del tiempo»:

"A good key for understanding how messianic (and how little apocalyptic) this end is and what kind of messianism is contained in Kojevian thought, can be found in what can already be called a contemporary classic on Messianism, Jacob Taubes' The Political Theology of Paul. Taubes, indeed, explains that in 1967 he invited Kojève to the University of Berlin for a lecture on Hegelian philosophy, where the latter stated that history had finished and all we can do thereafter is a repetition in an "as if" form (Taubes, 2004, p. 170). Repetition "as if" (history repeated only in its forms, without any historical content) is probably the key concept for understanding what kind of end of history is the Japanese one.» ${ }^{46}$

Y este «como si» kojeviano, por cierto, admite una comparación con el «als ob» de Adorno en Minima Moralia (Aingeru, 2009). Pero que admita tal comparación no significa necesariamente que se la pueda llevar demasiado lejos. Este nuevo formalismo ético à la Kojève es una relación con uno mismo (en el moralismo solitario) o con los demás (en el arte y la cultura) que sirve de impulso para la

42 KoJÈve, Esquisse d'une phénomenologie du droit, 1981, p. 216-218.

43 KoJÈve, Esquisse d'une phénomenologie du droit, 1981, p. 216.

44 KoJÈve, Introduction à la lecture de Hegel, 1968, p. 437.

45 DERRIDA, Specters of Marx: The state of the debt, the work of mourning and the new international, 1994, p. 71.

46 Aingeru, «The Japanese End: Japan in Alexandre Kojeve's End of History», 2009, p. 67. 
rememoración y el rescate del pasado en la Historia: una práctica humanizante ilimitada de recuperación de todas las figuras perecidas en la Historia, una suerte de redención figurada, «ritualizada».

En conclusión, la referencia a Japón, el «Imperio de los Signos» en palabras de Roland Barthes dos años después de la segunda edición de la Introduction à la lecture de Hegel (Aingeru, 2009), entraña no tanto un cambio de perspectiva hacia la filosofía oriental como una contextualización de la "negatividad sin empleo» de Bataille. Sans emploi, en efecto. Se trata de comprender bien lo siguiente: la acción posthistórica en este «tiempo del fin» rompe con el paradigma de la obra. Es, pues, antidialéctico, en la medida en que presupone la falta de una síntesis, una negatividad sin reconciliación posible y un sujeto abierto (no sujeto a nada ni a nadie). Lo que Kojève ha querido pensar es una forma de acción que siempre queda irresuelta, inoperante, desobrada. La mejor descripción de esta condición nos la da Kojève mismo en una extensa nota a pie de página de la Esquisse d'une phénomenologie du droit donde habla justamente del amor:

"[L'homme] Il se construit comme on construit une maison: il n'est vraiment humain que dans l'acte de se construire, mais le construit est un être non naturel, tout comme la maison. C'est le "caractère", la "personnalité» qui sont, comme sont les choses. On peut donc aimer non seulement l'animal proprement dit dans l'homme (son corps et son comportement physique et "psychique» animal), mais encore son être humain, c'est-à-dire le «cadavre» et la «momie» de ses actes anthropogènes. Ainsi on peut aimer l'homme mort dans la totalité de sa vie humaine écoulée, qui est "éternellement» (mais non en acte). On peut donc aimer le "caractère», la "personnalité» en se rapportant à l'homme pris dans son être et non dans son action. Mais il ne faut pas oublier que le "caractère» n'est vraiment humain (en acte) que dans la mesure où l'homme le «nie dialectiquement» (cette «négation» n'étant d'ailleurs réelle en acte que dans la mesure où elle crée une réalité, c'est-à-dire s'insère dans l'être et devient un être, c'est-à-dire s'inscrit dans un «caractère» et devient un "caractère» — d'où l'impossibilité et l'illusion du «romantisme» et la «révolution permanente»: il faut du temps pour réaliser une négation et pouvoir ainsi la nier à nouveau, et l'homme est mortel). On peut donc aimer l'humain dans l'homme, mais l'humain qu'on aime, étant être et non plus action, n'est plus humain en acte: c'est le souvenir de l'homme qu'on aime, même dans l'homme présent." 47

Esta negación continua es la posibilidad abierta de formar, conformar y reformar la propia naturaleza, y esto mismo es lo que está en juego en el esnobismo japonés de la segunda nota. Se trata de la apertura característica del paradigma postmetafísico; esto es, de la distinción entre «être» y «action» y de su imposible síntesis unificadora en un plano superior. Para expresarlo en lenguaje kantiano, es "la condición de posibilidad» para toda ética digna de este nombre. Es todo lo contrario a la animalización (Darwin), por un lado, y a la acción histórica (Hegel), por el otro. Lo snob es ético en la medida en que es prolegómeno para la práctica y la reflexión éticas en nuestro presente. La exigencia de separación entre forma (acción) y contenido (carácter) cuadra sorprendentemente con el marco de las filosofías del «cuidado de sí» (Foucault)

47 KoJÈve, Esquisse d'une phénomenologie du droit, 1981, p. 495. 
o la «ejercitación» (Sloterdijk). Kojève apuesta por la ligereza como serio estilo de vida («la vida es una comedia; pero uno debe representarla seriamente», habría dicho a $\operatorname{Aron}^{48}$ ). Puede que finalmente en la filosofía de nuestro autor todo sea comedia: se opone a la seriedad en Schmitt (teología) o en Strauss (filosofía socrática), por poner los dos ejemplos de sus célebres debates. Es más, tanto el Sabio como la actualización terminal del Estado universal y homogéneo (dos caras de la misma moneda) son parodias antiéticas. Ahora bien, su materialización deberá ser resistida por todos los medios filosóficos a nuestro alcance. Cualquier retorno al fin de la historia (esto es, a la tesis de la primera nota) es apocalíptico en la medida en que desaparecerá la negatividad humana (= espiritualidad). Y conservar esta negatividad inoperante es lo más alejado del nihilismo en que moraba el último hombre nietzscheano ${ }^{49}$.

48 Aron, Memoires: 50 ans de réflexion politique, 1983, p. 99.

49 Leo Strauss acusa el «fin de la historia» kojeviano de ser «el estado del último hombre de Nietzsche.» (Strauss, On Tyranny. Revised and Expanded Edition. Including the Strauss-Kojève Correspondence, 2000, p. 208) Con el hiperbólico esnobismo japonés, Kojève estaría, pues, respondiendo simultáneamente a Bataille i Strauss. Pero por encima de todo, en tono jocoso, estaría proponiendo algo muy serio: una apertura del bíos más allá de la circularidad dialéctica (hegeliana) en la Historia, quizá en los términos de una cura sui necesariamente posthistórica. Por otro lado, Agamben concluye: «Kojève privilegia el aspecto de la negación y de la muerte, y parece no ver el proceso por el cual, en la modernidad, el hombre (o el Estado, para él) comienza en cambio a ocuparse de la propia vida animal, y la vida natural se convierte, sobre todo, en lo que está en juego en lo que Foucault llamó biopoder. Tal vez, el cuerpo del animal antropóforo (el cuerpo del siervo) es el resto no resuelto que el idealismo deja en herencia al pensamiento, y las aporías de la filosofía de nuestro tiempo coinciden con las aporías de este cuerpo irremediablemente tenso y dividido entre animalidad y humanidad.» (AGAMBEn, Lo abierto. El hombre y el animal, 2006, p. 28) Aquí cabrían dos objeciones. En primer lugar, no es para nada cierto que Kojève omita la cuestión biopolítica: «Or l'État est aussi en rapport avec l'Homo sapiens qui n'est qu'animal qu'avec un individu humain pris en tant qu'animal. Ainsi, les mésures de santé publique ont souvent en vue l'animal dans l'homme.» (KoJÈve, Esquisse d'une phénomenologie du droit, 1981, p. 140); y en segundo lugar, lo cual todavía es más importante, la «tensión y división entre animalidad y humanidad», lejos de ser una aporía, es una invitación a pensar por fin una "forma de vida» (ética) desde su propia (ausencia de) raíz, sin el peso de la herencia del idealismo (Hegel). Para la comparación entre Kojève y Agamben, ver: Prozorov, G. (2009). "Giorgio Agamben and the End of History: Inoperative Praxis and the Interruption of the Dialectic», en: European Journal of Social Theory, 12 (4), pp. 523-542; Liakos, A. (2013). "The End of History as a Liminality of the Human Condition: From Kojève to Agamben, en: Turda, M. (Ed.). Crafting Humans: From Genetics to Eugenics and Beyond. Taipei: National Taiwan University Press, pp. 63-70; BorovinSKI, T. (2014). «Soberanía, secularización y final de la historia en Agamben y Kojève», en: El Banquete de los Dioses. Revista de Filosofía y Teoría Política contemporáneas, 2 (3), pp. 238-265. La conservación de una negatividad activa incluso en el fin de la historia permite un espacio abierto para la libertad y la virtud, es decir, para la humanidad en sentido ético. Evidentemente, todo ello arranca del «dualismo ontológico» kojeviano (cf. nota 10). 


\section{BibliografÍA}

Agamben, G. (2006). Lo abierto. El hombre y el animal. Buenos Aires: Adriana Hidalgo editora. Aingeru, R. (2009). "The Japanese End: Japan in Alexandre Kojeve's End of History», en: International Journal of Area Studies, 3, pp. 63-69.

Arendt, H. (1958). The Human Condition. Chicago: The University of Chicago Press.

Arendt, H. (1968). Men in Dark Times, New York: Harcourt, Brace \& World.

ARon, R. (1990). Memoires: 50 ans de réflexion politique. Paris: Julliard.

Auffret, D. (1990). Alexandre Kojève. La philosophie, l'État, la fin de l'Histoire. Paris: Grasset.

Bataille, G. (1996). Lo que entiendo por soberanía. Barcelona: Paidós.

BLoom, A. (1991). Gigantes y enanos. Barcelona: Gedisa.

Darby, T. (1990). The Feast: Meditations on Politics and Time. Toronto: University of Toronto Press.

Deblin, F. R. (2004). Alexandre Kojève and the Outcome of Modern Thought. Lanham, Maryland: University Press of America.

DERRIDA, J. (1994). Specters of Marx: The state of the debt, the work of mourning and the new international. New York: Routledge.

De VRIES, E. (2001). «Alexandre Kojève - Carl Schmitt Correspondence and Alexandre Kojève, "Colonialism from a European Perspective", en: Interpretation, 29/1, pp. 91-130.

Esouirol, J. M. (2006). "Alexandre Kojève, o com es distreu un hegelià al segle XX», en: Vergés, J. (Ed.). Cons i neocons: El referons filosòfic. Girona: Documenta.

KoJÈve, A. (1968). Introduction à la lecture de Hegel. Paris: Gallimard.

KoJÈve, A. (1981). Esquisse d'une phénomenologie du droit. Paris: Gallimard.

KoJÈve, A. (2006). La noción de Autoridad. Buenos Aires: Nueva Visión.

Murphy, G. (2011). "Alexandre Kojève: Cosmpolitanism at the End of History», en: Lee Trepanier \& Khalil M. Habib (Eds.), Cosmopolitanism in the Age of Globalization: Citizens without States. Lexington: University Press of Kentucky.

Rosen, S. (1987). Hermeneutics as Politics. Oxford: Oxford University Press.

Strauss, L. (2000). On Tyranny. Revised and Expanded Edition. Including the Strauss-Kojève Correspondence. (Edited by Victor Gourevitch and Michael S. Roth). Chicago: University of Chicago Press.

Departamento de Filosofía

ORIOL FARRÉs JuSte

Universidad Autónoma de Barcelona

Oriol.Farres@uab.cat

[Artículo aprobado para publicación en enero de 2018] 